\title{
Review Article \\ Repetitive Sequence and Sex Chromosome Evolution in Vertebrates
}

\author{
Tariq Ezaz and Janine E. Deakin \\ Institute for Applied Ecology, University of Canberra, Canberra, ACT 2601, Australia \\ Correspondence should be addressed to Tariq Ezaz; ezaz.tariq@gmail.com
}

Received 14 May 2014; Accepted 3 September 2014; Published 11 September 2014

Academic Editor: Paul Harrison

Copyright (C) 2014 T. Ezaz and J. E. Deakin. This is an open access article distributed under the Creative Commons Attribution License, which permits unrestricted use, distribution, and reproduction in any medium, provided the original work is properly cited.

\begin{abstract}
Sex chromosomes are the most dynamic entity in any genome having unique morphology, gene content, and evolution. They have evolved multiple times and independently throughout vertebrate evolution. One of the major genomic changes that pertain to sex chromosomes involves the amplification of common repeats. It is hypothesized that such amplification of repeats facilitates the suppression of recombination, leading to the evolution of heteromorphic sex chromosomes through genetic degradation of $\mathrm{Y}$ or $\mathrm{W}$ chromosomes. Although contrasting evidence is available, it is clear that amplification of simple repetitive sequences played a major role in the evolution of $\mathrm{Y}$ and $\mathrm{W}$ chromosomes in vertebrates. In this review, we present a brief overview of the repetitive DNA classes that accumulated during sex chromosome evolution, mainly focusing on vertebrates, and discuss their possible role and potential function in this process.
\end{abstract}

\section{Introduction}

Two major types of sex chromosome systems exist in vertebrates, XX female/XY male (e.g., human and salmon) and ZZ male/ZW female (birds and snakes). How these functionally important chromosomes evolve has been a topic for debate for more than a century, since the discovery of the first sex chromosomes in the late 1800s [1-3]. Despite the interest in this area, difficulties in sequencing highly repetitive $\mathrm{Y}$ and $\mathrm{W}$ chromosomes have hampered progress towards gaining a fuller understanding of the mechanisms involved in their evolution. This has resulted in the most detailed research on vertebrate sex chromosomes being carried out on species which have had at least part of the euchromatic region of their Y or W chromosome sequenced, for example, the evolutionary old sex chromosomes of eutherian mammals (three primates and two carnivores) [4-7] or the evolutionarily young sex chromosomes of fishes such as the halfsmooth tongue sole [8], three-spine stickleback [9], and medaka [10]. The comparison between species of evolutionarily advanced $Y$ chromosomes is not ideal for gaining insight into the mechanisms driving sex chromosome evolution as the chromosomes have undergone extensive changes and degeneration, perhaps even losing key clues required to unravel their evolution. In contrast, the sequencing of young sex chromosomes should help in elucidating these driving mechanisms [11], particularly if comparisons can be made to more highly diverged sex chromosomes that share a common ancestry.

Although we have some understanding of the molecular organization of sex chromosomes in model vertebrate species, it is largely unknown for the majority of the species where sex chromosomes have been identified cytologically. The conservation of sex chromosome gene content and the sex determining gene in most mammals does not reflect the diversities that exist in other vertebrate groups, where there has been rapid evolution of sex chromosomes in many lineages [12]. Despite the cytogenetic identification of nonhomologous sex chromosomes among vertebrates, very few $\mathrm{Y}$ or W chromosomes have been sufficiently mapped and/or sequenced, for studies into their evolution, largely due to the abundant repetitive sequences on these chromosomes [13], and only a few sex determining genes have been identified. Nonetheless, the latest advances in molecular cytogenetics, DNA sequencing, and bioinformatics are making it possible to study the make-up of sex chromosomes in greater detail 
than ever before. Here we review the association of the amplification of repetitive sequences near the sex determining locus and discuss their possible role in the evolution of sex chromosomes and their potential function. As the technical advances in this field are relatively recent, we call upon data from organisms outside the vertebrate phylogeny to identify important areas for future research in vertebrates.

\section{Sex Chromosome Evolution in Vertebrates}

Sex chromosomes have evolved multiple times and independently throughout evolution. They have many unique features, including unique gene content (e.g., sex linked genes, including the master sex determining genes, such as $S R Y$ in most mammals) and existence of highly variable morphology among different taxa, often representing various evolutionary stages [14-16]. Such morphological variations have formed due to a suppression of recombination leading to gene loss and the accumulation of repetitive sequence on one of the homologues (Y or W) $[14,17,18]$. They also experience special selective pressures compared to those of autosomes which, although debatable, are proposed to include both positive and negative selections, such as purifying selection to maintain sequences in the $\mathrm{X}$-degenerate regions on the human Y chromosome [19] and even sex-specific selection, which has been demonstrated to impact on $\mathrm{W}$ chromosome gene expression in chicken [20]. These unique features make sex chromosomes the most dynamic entity in any genome $[15,17,21]$, providing unique opportunities to study and understand genome evolution and organization.

Vertebrate sex chromosomes display enormous diversity in morphology and in gene content [12, 17, 22-27]. Such diversities not only imply multiple and independent origins of sex chromosomes, but also suggest evolution of a very specific molecular mechanism that is uniquely dynamic in performing a very specific task-sex determination. Despite such enormous diversities among taxa, two competing hypotheses have been put forward on sex chromosome evolution and degeneration based on the studies derived from mammals and birds ( $\mathrm{Y}$ and $\mathrm{W}$ degeneration) and from nonamniotes, such as fish and frogs (fountain of youth) [15, 18, 28-31].

In most mammals, sex chromosomes are highly differentiated morphologically, usually represented by a large and gene rich $\mathrm{X}$ chromosome and a small, degenerated, and gene poor Y chromosome. This heteromorphism and subsequent in-depth molecular analysis led to the postulation of the most accepted theory for sex chromosome evolution. This hypothesis posits that sex chromosomes have evolved from an autosomal pair when the proto-Y chromosome acquired a sex determining gene/locus [28, 29]. Subsequently, chromosome rearrangements including accumulation of other sexually advantageous genes near the sex determining locus drove selection for suppression of recombination. This process in turn facilitated loss of active genes, deletions, and insertions, leading to degeneration of the proto-Y, making the sex chromosome pair morphologically differentiated [14, 18, 29, 32]. This hypothesis considers suppression of recombination as a result of chromosome rearrangements, which gradually expand into the nonrecombining regions leading to the gradual loss of genes from Y chromosomes, leaving only sets of genes that are required for development of testes and brain development [33]. It is well known that chromosome rearrangements (such as inversions, insertions, and deletions) suppress recombination between homologous chromosomes $[34,35]$. The best example would include differentiation of mammalian sex chromosomes which has occurred through the genetic degradation of the Y chromosome following rearrangements $[17,23]$. Although the chicken $\mathrm{W}$ chromosome appears to have evolved independently, it is proposed to have followed the same mechanisms [15].

Despite the tremendous morphological diversities of sex chromosomes observed in amniotes, the scenario is quite the opposite in nonamniotes, such as in fish and amphibians. Although most of the fish and amphibians (so far studied) display genotypic sex determination (GSD), in the majority of species, sex chromosomes are cryptic; that is, they are not morphologically differentiated [36-39]. Evolution of such homomorphic sex chromosomes in fish and amphibians has been attributed to the rapid turnover of sex chromosomes through acquisition of sex chromosome functions by native/existing genes, often unrelated to those that are part of the sex differentiating cascade $[26,30,40,41]$. The mechanism behind the evolution of such rapid turnover through de novo evolution of sex determining genes is not fully understood. However, Perrin [31] argued that such a mechanism is likely to be maintained by sex reversal and occasional recombination between sex chromosomes. The author's observation is based on the empirical data that the recombination frequencies are associated with the phenotypes rather than the genotypes. Therefore, because the sex chromosomes are not strictly recombinationally isolated from each other (i.e., recombination in sex reversed individuals, which is common in fish and amphibians), the $\mathrm{Y} / \mathrm{W}$ are protected from degradation (fountain of youth). Certainly, more evidence is required to support this hypothesis and is likely to be published in coming years from studies on fish to reptiles.

\section{Chromosome Rearrangements and Repeat Accumulation}

It is a well-observed phenomenon that chromosome rearrangements occurred particularly adjacent to the sex determination locus in most taxa. These include deletions, insertions, inversions, transpositions, and amplification of repetitive sequences [42-50]. This may suggest that it is likely that the sex determining locus or gene arises in a region of a chromosome which is unstable or fragile (e.g., contains common classes of fragile sites, such as AT-rich) or in a region which allows chromatin modification through cellular mechanisms (e.g., histone modifications). However, this is certainly not the case in mammalian sex chromosomes, as the human $\mathrm{X}$ chromosome contains only three fragile sites, while the Y contains none [46]. Nonetheless, the sex determining gene $S R Y$ lies very close to the pseudoautosomal region (PAR), which seems to be somewhat unstable [51]. However, the absence of fragile sites may represent advanced sex chromosomes, which have gone through the evolutionary 


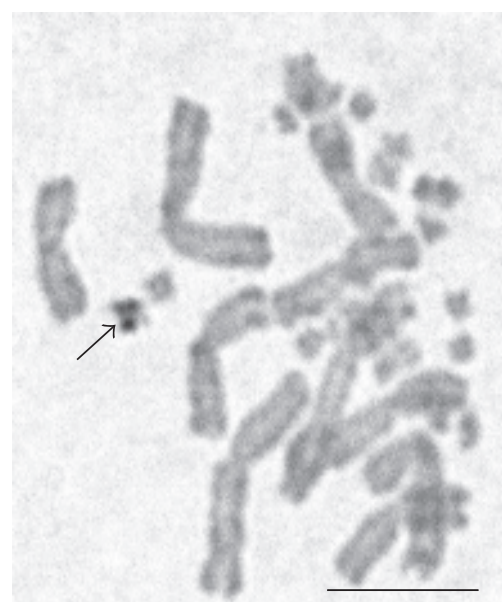

(a)

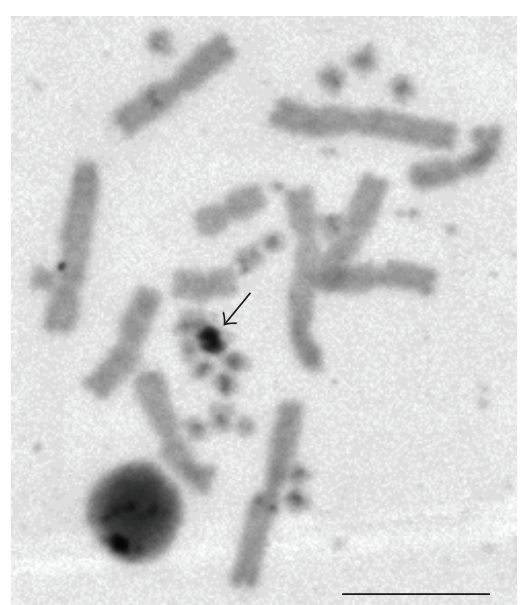

(b)

FIGURE 1: C-banding in Australian dragon lizards showing accumulation of heterochromatin on W chromosome: (a) Ctenophorus fordi, (b) Pogona vitticeps. Note that the ZW sex chromosomes between these two species are nonhomologous [12], yet the heterochromatin accumulation on the $\mathrm{W}$ chromosomes is very similar. For more images see [42, 43]. Arrows indicate $\mathrm{W}$ chromosomes. Scale bars represent $10 \mu \mathrm{M}$.

process of stabilization, but the presence of fragile sites may be a common phenomenon in many animals and plants with nascent sex chromosomes. It may also be likely that the sex determining locus or gene destabilizes the chromosome region in which it is located.

Simple repetitive sequences (e.g., microsatellites) are often accumulated in high copy numbers on the sex chromosomes in many taxa [41-45, 52-54], although those same motifs have low copy numbers distributed throughout the genome, implying preferential amplification. Even standard cytogenetic technique, such as C-banding, is able to detect heterochromatin accumulation on sex chromosomes (Figure 1). Why repetitive sequences preferentially amplify on sex chromosomes is still unknown, yet many theories have been put forward. One of the well-accepted theories is that the accumulation of repetitive sequences on one of the pair of sex chromosomes facilitates suppression of recombination between sex chromosome homologues, therefore, protecting the sexually beneficial mutations $[14,15]$. On the other hand, it is equally plausible that chromosome rearrangements as well as repeat accumulation and amplification may occur near the sex determining locus as a result of suppression of recombination, rather than inducing it [55-57].

There have been a limited number of studies on relatively young sex chromosomes that have shown that the suppression of recombination, repeat accumulation, and chromosomal rearrangement can occur rapidly. In medaka (Oryzias latipes), the sex determining gene $d m r t 1 b Y$ is derived from a duplicated fragment of an autosome that has inserted onto the proto-Y chromosome [10]. Sequence data indicates that repeats expanded in the sex determining region after the insertion onto the proto-Y [10]. Sequence comparisons and gene mapping of the homomorphic $\mathrm{X}$ and Y chromosomes of the three-spine stickleback (Gasterosteus aculeatus) have demonstrated that even these supposedly nascent sex chromosomes have diverged considerably, with higher repeat content on the $\mathrm{Y}$ than the $\mathrm{X}$ and chromosomal rearrangements such as inversions and deletion $[9,58]$. However, in both of these cases, it is still unclear whether repeat accumulation led to suppressed recombination or vice versa.

While likely molecular mechanisms behind chromosome rearrangements are reasonably well known, the alternate mechanisms that initiate suppression of recombination near the sex determining locus are yet to be elucidated. It is also possible that a heritable epimutation, such as a change in DNA methylation, and not a genetic mutation in the sex determining locus may be the first step in sex chromosome evolution $[59,60]$. If this epimutation could suppress recombination, it may result in the region being more susceptible to genetic mutation $[59,61]$ and the insertion of repetitive elements. One plausible and testable hypothesis behind suppression of recombination near the sex determining locus would lie within chromosome architecture. This primarily includes changes in chromatin structure (epigenetic such as DNA methylation and/or histone modification) inflicted by a sexspecific mutation, which is sufficient to suppress or reduce recombination between sex chromosomes. Investigation of chromosomal architecture around the region adjacent to a putative sex determining locus in nascent sex chromosomes from various taxa, including birds and snakes (e.g., ratite birds and boid snakes) would provide empirical evidence for unraveling any mechanisms which may be epigenetically driven.

Studies on the formation of the accumulation of repeats on the Y chromosomes of Drosophila species are perhaps more advanced than they are for vertebrates and provide valuable insight for future studies on the evolution of vertebrate sex chromosomes. By comparing Drosophila species with neo-sex chromosomes of different ages, it has been 
TABLE 1: An overview of repeats on sex chromosomes among divergent taxa.

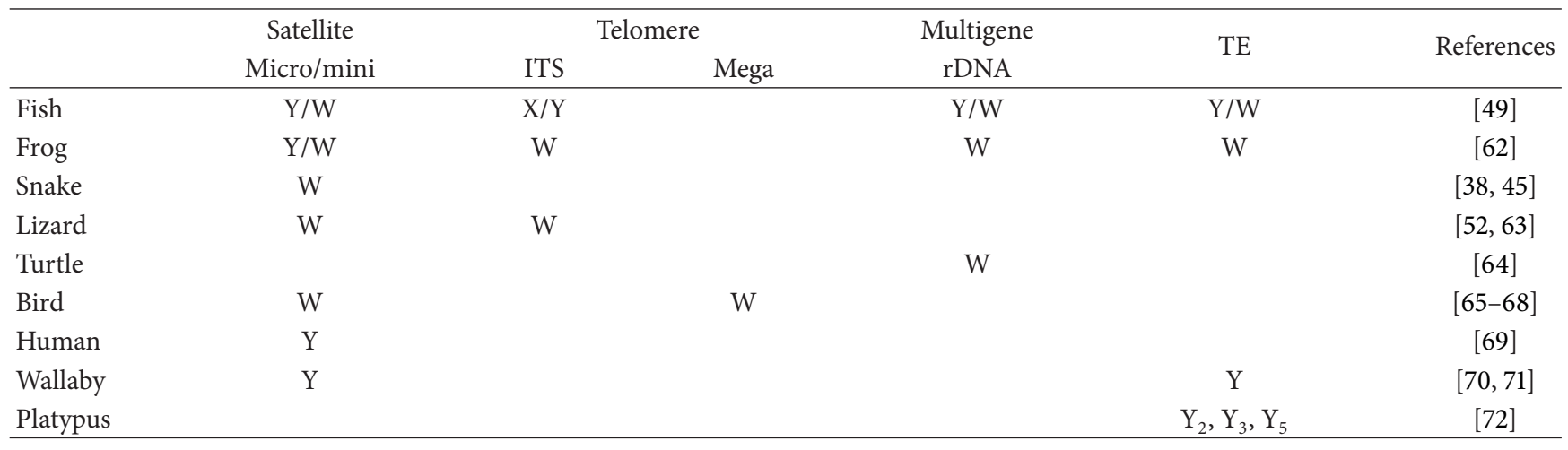

(ITS: interstitial telomere signal; TE: transposable elements).

possible to start tracing the events leading to the formation of a heterochromatic Y chromosome [73-76]. For instance, $D$. miranda, neo-sex chromosomes were formed approximately 1 million years ago (MYA) from the fusion of the ancestral $\mathrm{Y}$ chromosome with an autosome. This neo-Y chromosome presents a case of $\mathrm{Y}$ chromosome degradation and repeat sequence accumulation caught in the act, with only 1941 of the 2951 neo-X genes possessing intact open reading frames on the neo- $Y$ and with almost half of these neo- $Y$ genes expressed at lower levels than their neo-X counterparts [75]. In addition, nearly $50 \%$ of the neo-Y sequence consists of repeats $[77,78]$, demonstrating the rapid changes that have occurred in the evolution of sex chromosomes in just 1 million years of evolution [73]. Interestingly, the neo$\mathrm{Y}$ is also enriched for epigenetic marks associated with heterochromatin, such as H3K9me2 and HP1 $\alpha$. Furthermore, neo-Y regions with higher repeat content have higher levels of $\mathrm{H} 3 \mathrm{~K} 9 \mathrm{me} 2$ binding, which is consistent with models proposing that repeat accumulation enables the formation of heterochromatin on nascent Y or W chromosomes [76]. It is to be hoped that similar studies into the sequence and epigenetic features of vertebrate sex chromosomes will not be too far behind these Drosophila studies. Further studies involving high resolution sequence analysis of sex determining region in vertebrates, as well as investigation of chromosomal architecture surrounding the sex determining region, will reveal the true mechanisms that drive sex chromosome evolution after acquisition of a sexually advantageous gene or locus.

\section{Is There a Particular Class (or Classes) of Repeats That Amplified Preferentially on Sex Chromosomes?}

Large volumes of research papers have been published on physical mapping of various repetitive sequences in divergent taxa, from plants to mammals. These include amplification of satellite DNA (mini and micro), telomeric sequences (including megatelomere in chicken), amplification of multigene families (rDNA and histones), taxon specific repeats, transposable elements (LINEs and SINEs), and multicopy genes. Table 1 presents a representative summary of the various classes of repetitive sequences which have been mapped in vertebrates. However, the majority of the mapping has been done on fishes as a tool for identifying sex chromosomes, as their sex chromosomes are often homomorphic. Amphibians also have a high frequency of homomorphic sex chromosomes; however, there have been only limited studies where repetitive sequences have been used to identify sex chromosomes [62]. The minisatellite repeat Bkm (branded krait minor) was characterized on the $\mathrm{W}$ chromosome of a snake species in the early 70s [79] and in many other species including snakes [80-85]. Only two studies have so far been published on the repeat content of lizard sex chromosomes [52,63]. It may not be the true scenario because of the unavailability of mapping information of all repetitive classes in representative taxa, but overwhelming data on the amplification of simple repeats near the sex-determining locus in divergent taxa suggest a common trend; that is, such accumulation of simple repeats may not be an artifact of the volume of the published literature but a true representation of the molecular mechanism. Information from sequenced $\mathrm{Y}$ and $\mathrm{W}$ chromosomes would support this as, for example, the half-smooth tongue sole $\mathrm{W}$ has more than double the repeat content of $\mathrm{Z}$ [8]. Perhaps this convergent amplification of simple repeats triggered by sex-linked mutation is the prime genomic driver that initiates sex chromosome evolution in many taxa.

\section{Do Sex Chromosome Repeats Have a Function?}

In the past, repetitive sequences were dismissed as part of the "junk DNA" [86], a term used by Ohno to describe the non-protein-coding regions of the genome [87]. We now know that at least some of these sequences are transcribed and obviously play a functional role in the genome [86]. When it comes to sex chromosomes, the idea of "junk DNA" is also gradually being rejected. Recent findings in taxonomically diverse species suggest that these sequences play an important role. However, evidence of a functional role of repeats on vertebrate sex chromosomes is limited at this stage, largely due to the difficulty in obtaining $\mathrm{Y}$ 
or $\mathrm{W}$ chromosome sequences. Nonetheless, methods have been developed to achieve this challenging task $[88,89]$ and will undoubtedly lead to more studies in this area. Again, we are able to gain important insight into the function of sex chromosome repeats from species outside the vertebrate lineage.

One elegant study on the function of sex chromosome repeats was carried out on Schistosoma mansoni, a parasitic platyhelminth, with a ZW sex determination system. The $\mathrm{Z}$ and $\mathrm{W}$ chromosomes can be cytogenetically distinguished by their chromatin structure rather than by their size. Sequencing of male and female genomes led to the identification of $\mathrm{W}$-specific sequences, which consisted of $36 \mathrm{~W}$-specific repeats, including an SMalpha retroposon, LTRs, LINE2, and DNA transposons [89]. Of these repeats, transcription was detected for eight, and three of these were transcribed in the larval stages but not in immature or adult females. Using chromatin immunoprecipitation sequencing (ChIP-Seq), Lepesant and colleagues [89] were able to profile the level of an epigenetic mark associated with active chromatin, acetylated $\mathrm{H} 3 \mathrm{~K} 9(\mathrm{H} 3 \mathrm{~K} 9 \mathrm{Ac})$, around repeats at various developmental stages. A gradual decrease in the level of H3K9Ac was observed for all $\mathrm{W}$-specific repeats from larval to adult stages. Subsequent experiments performed on two transcribed and one nontranscribed repeats showed enrichment for another active mark, trimethylated H3K4 (H3K4Me3), in larval stages compared to adults. Repressive marks trimethylated $\mathrm{H} 3 \mathrm{~K} 9$ (H3K9Me3) and trimethylated H3K27 (H3K27Me3) associated with heterochromatin were enriched at these three repeats in cercariae, the larval stage capable of infecting a vertebrate host, but less abundant in miracidia (a different larval stage) and adults. It appears that the transcription of these repeats can be correlated with changes in chromatin structure. Lepesant and colleagues [89] suggest that it may even be these changes in chromatin structure that may contribute to sex determination, perhaps even in the absence of a sex determining gene.

In Drosophila, the Y chromosome consists of megabase regions of repetitive sequences, such as microsatellites, transposable elements, and ribosomal DNA (rDNA), and it has been hypothesized that variation in the type, abundance, and distribution of these repetitive sequences can influence gene expression across the genome [90]. Most Drosophila $\mathrm{Y}$ chromosome polymorphisms are not located in proteincoding genes but in the heterochromatic regions where repetitive sequences are abundant. These polymorphisms affect the expression of many autosomal and X-linked genes [9194], typically those that are located in repressive chromatin and are subject to tissue-specific expression [90]. It would appear that the $\mathrm{Y}$ chromosome acts as a giant regulator of gene expression through its global effects on chromatin dynamics $[92,95-97]$ and there is evidence from male and XXY female Drosophila lines, differing only in the origin of their Y chromosomes, that there is a yet to be ascertained underlying epigenetic mechanism involved [93]. This gene regulator is not acting by turning genes on or off but by working at a more subtle scale of gene regulation [98]. This effect on gene expression is also observed in XXY females where Y-linked genes are not expressed, providing additional support for the idea that the heterochromatic region of the $\mathrm{Y}$ acts as a gene expression modulator [93].

It would be interesting to know if the heterochromatic region of the human Y chromosome similarly plays a role in gene regulation. Such studies are yet to be conducted on the human Y, as most research efforts have focused on the protein-coding genes and not the heterochromatic region [98]. However, copy number variation of genes present in multiple copies on the mouse Y chromosome (Sly and Rmby) is correlated with regulation of autosomal immune gene expression and, similar to the Drosophilia Y, is most likely the result of a chromatin remodeling mechanism, suggesting that this regulatory function of the Y chromosome may be widespread [99]. Furthermore, epigenetic profiling of tandem repeat sequences within the euchromatic region of the human $Y$ has shown specific patterns of histone modifications (active mark H3K9Ac, repressive marks H3K9me3 and H3K27me3) and CTCF binding (transcriptional repressor) associated with different repeats. These specific patterns of epigenetic marks suggest that tandem repeats on the $\mathrm{Y}$ chromosome may play a role in chromatin status and may act as regulatory elements [100].

The Bkm satellite repeat consists of tandem arrays of GATA nucleotides. Although not necessarily specific to the sex chromosomes, Bkm repeats are abundant on heterogametic sex chromosomes of divergent vertebrate species [81]. A sex and tissue-specific protein that binds specifically to $\mathrm{Bkm}$ repeats known as Bkm-binding protein (BBP) appears to be involved in coordinated decondensation of the heterogametic sex chromosome in germ cells [101, 102]. Subramanian et al. [103] reported that the GATA repeats on the human Y chromosome may play a role in marking the boundaries of chromatin domains. More recently, it has been discovered that GATA repeats have a conserved role, acting as insulators in both Drosophila and human cells [104].

Determining the function of repetitive sequences on $\mathrm{Y}$ or $\mathrm{W}$ chromosomes is in its infancy but it is clear that these sequences do play a functional role in gene regulation and chromatin structure. The demonstrated ability of the Drosophila and mouse Y chromosome to regulate gene expression across the genome is particularly exciting and is an area of research that needs to be pursued in more vertebrate species.

\section{Conclusions/Future Directions}

It is clear that repetitive sequences are a prominent feature of sex chromosomes across plant and animal kingdoms. These repeats have proven challenging for efforts to sequence $Y$ and $\mathrm{W}$ chromosomes but bioinformaticians are rising to the challenge and are developing methods to obtain sequences from these unique chromosomes. This will enable many more $\mathrm{Y}$ or W-specific repeats to be identified and their functions to be determined. Typically, the heterochromatic regions of these chromosomes, where repeats are abundant, are ignored in favour of studying euchromatic regions containing protein-coding genes. However, even the limited number of studies that have been carried out on $\mathrm{Y}$ and $\mathrm{W}$ repeat function has shown that these sequences play an important role in gene 
regulation, even beyond that of the sex chromosome itself. By determining the function of these repeats, perhaps we can then decipher whether the accumulation of repeats on sex chromosomes is a cause or consequence of recombination suppression and gain a better understanding of the steps involved in sex chromosome evolution.

\section{Conflict of Interests}

The authors declare that there is no conflict of interests regarding the publication of this paper.

\section{Acknowledgments}

The authors would like to thank Ikuo Miura for his valuable comments on the paper. Tariq Ezaz and Janine E. Deakin are supported by ARC Future Fellowships (FT110100733, FT100100241).

\section{References}

[1] H. Henking, "Über Patagonian pejerrey (Odontesthes hatcheri) spermatogenese und deren Beziehung zur Entwicklung bei Pyrrhocoris apterus L," Zeitschrift für Wissenschaftliche Zoologie, vol. 51, pp. 685-736, 1891.

[2] N. M. Stevens, Studies in Spermatogenesis with Especial Reference to the "Accessory Chromosome", vol. 36, Carnegie Institution of Washington Publication, 1905.

[3] E. B. Wilson, "The chromosomes in relation to the determination of sex in insects," Science, vol. 22, pp. 500-502, 1905.

[4] J. F. Hughes, H. Skaletsky, L. G. Brown et al., "Strict evolutionary conservation followed rapid gene loss on human and rhesus y chromosomes," Nature, vol. 483, no. 7387, pp. 82-87, 2012.

[5] J. F. Hughes, H. Skaletsky, T. Pyntikova et al., "Chimpanzee and human y chromosomes are remarkably divergent in structure and gene content," Nature, vol. 463, no. 7280, pp. 536-539, 2010.

[6] G. Li, B. W. Davis, T. Raudsepp et al., "Comparative analysis of mammalian y chromosomes illuminates ancestral structure and lineage-specific evolution," Genome Research, vol. 23, no. 9, pp. 1486-1495, 2013.

[7] H. Skaletsky, T. Kuroda-Kawaguchl, P. J. Minx et al., "The malespecific region of the human $\mathrm{Y}$ chromosome is a mosaic of discrete sequence classes," Nature, vol. 423, no. 6942, pp. 825837, 2003.

[8] S. Chen, G. Zhang, C. Shao et al., "Whole-genome sequence of a flatfish provides insights into ZW sex chromosome evolution and adaptation to a benthic lifestyle," Nature Genetics, vol. 46, no. 3, pp. 253-260, 2014.

[9] C. L. Peichel, J. A. Ross, C. K. Matson et al., "The master sexdetermination locus in threespine sticklebacks is on a nascent Y chromosome," Current Biology, vol. 14, no. 16, pp. 1416-1424, 2004.

[10] M. Kondo, U. Hornung, I. Nanda et al., "Genomic organization of the sex-determining and adjacent regions of the sex chromosomes of medaka," Genome Research, vol. 16, no. 7, pp. 815-826, 2006.

[11] D. Charlesworth, B. Charlesworth, and G. Marais, "Steps in the evolution of heteromorphic sex chromosomes," Heredity, vol. 95, no. 2, pp. 118-128, 2005.
[12] T. Ezaz, S. D. Sarre, D. O’Meally, J. A. Marshall Graves, and A. Georges, "Sex chromosome evolution in lizards: independent origins and rapid transitions," Cytogenetic and Genome Research, vol. 127, no. 2-4, pp. 249-260, 2010.

[13] A. Bernardo Carvalho and A. G. Clark, "Efficient identification of y chromosome sequences in the human and drosophila genomes," Genome Research, vol. 23, no. 11, pp. 1894-1907, 2013.

[14] J. J. Bull, Evolution of Sex Determining Mechanisms, Benjamin/Cummings Publishing Company, Menlo Park, Calif, USA, 1983.

[15] S. Ohno, Sex Chromosomes and Sex-Linked Genes, Springer, Heidelberg, Germany, 1967.

[16] A. J. Solari, Sex Chromosomes and Sex Determination in Vertebrates, CRC Press, Boca Raton, Fla, USA, 1993.

[17] B. Charlesworth, "The evolution of sex chromosomes," Science, vol. 251, no. 4997, pp. 1030-1033, 1991.

[18] W. R. Rice, "Evolution of the Y sex chromosome in animals," BioScience, vol. 46, no. 5, pp. 331-343, 1996.

[19] J. F. Hughes, H. Skaletsky, T. Pyntikova et al., "Conservation of Y-linked genes during human evolution revealed by comparative sequencing in chimpanzee., Nature, vol. 437, no. 7055, pp. 100-103, 2005.

[20] H. K. Moghadam, M. A. Pointer, A. E. Wright, S. Berlin, and J. E. Mank, "W chromosome expression responds to female-specific selection," Proceedings of the National Academy of Sciences of the United States of America, vol. 109, no. 21, pp. 8207-8211, 2012.

[21] H. J. Muller, "A gene for the fourth chromosome of Drosophila," Journal of Experimental Zoology, vol. 17, pp. 325-336, 1914.

[22] T. Ezaz, R. Stiglec, F. Veyrunes, and J. A. Marshall Graves, "Relationships between vertebrate ZW and XY sex chromosome systems," Current Biology, vol. 16, no. 17, pp. R736-R743, 2006.

[23] J. A. M. Graves, "Sex chromosome specialization and degeneration in mammals," Cell, vol. 124, no. 5, pp. 901-914, 2006.

[24] J. E. Mank, "Small but mighty: the evolutionary dynamics of W and y sex chromosomes," Chromosome Research, vol. 20, no. 1, pp. 21-33, 2012.

[25] I. Miura, H. Ohtani, and M. Ogata, "Independent degeneration of W and y sex chromosomes in frog Rana rugosa," Chromosome Research, vol. 20, no. 1, pp. 47-55, 2012.

[26] D. O'Meally, T. Ezaz, A. Georges, S. D. Sarre, and J. A. M. Graves, "Are some chromosomes particularly good at sex? Insights from amniotes," Chromosome Research, vol. 20, no. 1, pp. 7-19, 2012.

[27] S. D. Sarre, T. Ezaz, and A. Georges, "Transitions between sex-determining systems in reptiles and amphibians," Annual Review of Genomics and Human Genetics, vol. 12, pp. 391-406, 2011.

[28] R. Fisher, “The evolution of dominance," Biological Reviews, vol. 6, no. 4, pp. 345-368, 1931.

[29] B. Charlesworth and D. Charlesworth, "The degeneration of Y chromosomes," Philosophical Transactions of the Royal Society B: Biological Sciences, vol. 355, no. 1403, pp. 1563-1572, 2000.

[30] J. A. M. Graves and C. L. Peichel, "Are homologies in vertebrate sex determination due to shared ancestry or to limited options?" Genome Biology, vol. 11, no. 4, article 205, 2010.

[31] N. Perrin, "Sex reversal: a fountain of youth for sex chromosomes?" Evolution, vol. 63, no. 12, pp. 3043-3049, 2009.

[32] M. Nei, "Linkage modifications and sex difference in recombination.," Genetics, vol. 63, no. 3, pp. 681-699, 1969.

[33] J. A. M. Graves, "Review: sex chromosome evolution and the expression of sex-specific genes in the placenta," Placenta, vol. 31, pp. S27-S32, 2010. 
[34] D. Dumas and J. Britton-Davidian, "Chromosomal rearrangements and evolution of recombination: comparison of chiasma distribution patterns in standard and Robertsonian populations of the house mouse," Genetics, vol. 162, no. 3, pp. 1355-1366, 2002.

[35] C. O. Ostberg, L. Hauser, V. L. Pritchard, J. C. Garza, and K. A. Naish, "Chromosome rearrangements, recombination suppression, and limited segregation distortion in hybrids between Yellowstone cutthroat trout (Oncorhynchus clarkii bouvieri) and rainbow trout (O. mykiss)," BMC Genomics, vol. 14, no. 1, article 570, 2013.

[36] R. H. Devlin and Y. Nagahama, "Sex determination and sex differentiation in fish: an overview of genetic, physiological, and environmental influences," Aquaculture, vol. 208, no. 3-4, pp. 191-364, 2002.

[37] C. Eggert, "Sex determination: the amphibian models," Reproduction Nutrition Development, vol. 44, no. 6, pp. 539-549, 2004.

[38] M. Schartl, "Sex chromosome evolution in non-mammalian vertebrates," Current Opinion in Genetics and Development, vol. 14, no. 6, pp. 634-641, 2004.

[39] M. Schmid, I. Nanda, C. Steinlein, K. Kausch, T. Haaf, and J. T. Epplen, "Chapter 16-sex-determining mechanisms and sex chromosomes in amphibia," in Amphibian Cytogenetics and Evolution, D. M. Green and S. K. Sessions, Eds., pp. 393-430, Academic Press, San Diego, Calif, USA, 1991.

[40] O. Blaser, C. Grossen, S. Neuenschwander, and N. Perrin, "Sexchromosome turnovers induced by deleterious mutation load," Evolution, vol. 67, no. 3, pp. 635-645, 2013.

[41] M. B. Cioffi and L. A. C. Bertollo, "Chromosomal distribution and evolution of repetitive DNAs in Fish," Genome Dynamics, vol. 7, pp. 197-221, 2012.

[42] K. Matsubara, T. Knopp, S. D. Sarre, A. Georges, and T. Ezaz, "Karyotypic analysis and FISH mapping of microsatellite motifs reveal highly differentiated $\mathrm{XX} / \mathrm{XY}$ sex chromosomes in the pink-tailed worm-lizard (Aprasia parapulchella, Pygopodidae, Squamata)," Molecular Cytogenetics, vol. 6, no. 1, article 60, 2013.

[43] K. Matsubara, S. D. Sarre, A. Georges, Y. Matsuda, J. A. Marshall Graves, and T. Ezaz, "Highly differentiated ZW sex microchromosomes in the australian varanus species evolved through rapid amplification of repetitive sequences," PLOS ONE, vol. 9, no. 4, Article ID e95226, 2014.

[44] M. B. Cioffi, J. P. M. Camacho, and L. A. C. Bertollo, "Repetitive DNAs and differentiation of sex chromosomes in neotropical fishes," Cytogenetic and Genome Research, vol. 132, no. 3, pp. 188-194, 2011.

[45] M. B. Cioffi, E. Kejnovsky, and L. A. C. Bertollo, "The chromosomal distribution of microsatellite repeats in the genome of the wolf fish Hoplias malabaricus, focusing on the sex chromosomes," Cytogenetic and Genome Research, vol. 132, no. 4, pp. 289-296, 2011.

[46] S. G. Durkin and T. W. Glover, "Chromosome fragile sites," Annual Review of Genetics, vol. 41, pp. 169-192, 2007.

[47] L. D. Hurst and H. Ellegren, "Sex biases in the mutation rate," Trends in Genetics, vol. 14, no. 11, pp. 446-452, 1998.

[48] E. Olmo, "Reptilia," in Animal Cytogenetics Volume 4: Chordata, B. John, Ed., part 3, Gebrüder Borntraeger, Berlin, Germany, 1986.

[49] K. M. Reed and R. B. Phillips, "Polymorphism of the nucleolus organizer region (NOR) on the putative sex chromosomes of Arctic char (Salvelinus alpinus) is not sex related," Chromosome Research, vol. 5, no. 4, pp. 221-227, 1997.
[50] K. Matsubara, T. Gamble, Y. Matsuda et al., "Non-homologous sex chromosomes in two geckos (Gekkonidae: Gekkota) with female heterogamety," Cytogenetic and Genome Research. In press.

[51] F. Gianfrancesco, R. Sanges, T. Esposito et al., "Differential divergence of three human pseudoautosomal genes and their mouse homologs: Implications for sex chromosome evolution," Genome Research, vol. 11, no. 12, pp. 2095-2100, 2001.

[52] M. Pokorná, L. Kratochvíl, and E. Kejnovský, "Microsatellite distribution on sex chromosomes at different stages of heteromorphism and heterochromatinization in two lizard species (Squamata: eublepharidae: coleonyx elegans and lacertidae: eremias velox)," BMC Genetics, vol. 12, article 90, 2011.

[53] Z. Kubat, R. Hobza, B. Vyskot, and E. Kejnovsky, "Microsatellite accumulation on the Y chromosome in Silene latifolia," Genome, vol. 51, no. 5, pp. 350-356, 2008.

[54] J. Santos, L. Serra, E. Solé, and M. Pascual, "FISH mapping of microsatellite loci from drosophila subobscura and its comparison to related species," Chromosome Research, vol. 18, no. 2, pp. 213-226, 2010.

[55] R. Bergero, D. Charlesworth, D. A. Filatov, and R. C. Moore, "Defining regions and rearrangements of the Silene latifolia Y chromosome," Genetics, vol. 178, no. 4, pp. 2045-2053, 2008.

[56] M. Iwase, Y. Satta, Y. Hirai, H. Hirai, H. Imai, and N. Takahata, "The amelogenin loci span an ancient pseudoautosomal boundary in diverse mammalian species," Proceedings of the National Academy of Sciences of the United States of America, vol. 100, no. 9, pp. 5258-5263, 2003.

[57] G. Marais and N. Galtier, "Sex chromosomes: how X-Y recombination stops," Current Biology, vol. 13, no. 16, pp. R641-R643, 2003.

[58] J. A. Ross and C. L. Peichel, "Molecular cytogenetic evidence of rearrangements on the $\mathrm{Y}$ chromosome of the threespine stickleback fish," Genetics, vol. 179, no. 4, pp. 2173-2182, 2008.

[59] R. Gorelick, "Evolution of dioecy and sex chromosomes via methylation driving Muller's ratchet," Biological Journal of the Linnean Society, vol. 80, no. 2, pp. 353-368, 2003.

[60] E. Jablonka and M. J. Lamb, "The evolution of heteromorphic sex chromosomes," Biological Reviews of the Cambridge Philosophical Society, vol. 65, no. 3, pp. 249-276, 1990.

[61] E. Jablonka, "The evolution of the peculiarities of mammalian sex chromosomes: an epigenetic view," Bioessays, vol. 26, no. 12, pp. 1327-1332, 2004.

[62] M. Suda, Y. Uno, Y. Mori, Y. Matsuda, and M. Nakamura, "Molecular cytogenetic characterization of telomere-specific repetitive DNA sequences in Rana rugosa," Journal of Experimental Zoology A: Ecological Genetics and Physiology, vol. 315, no. 4, pp. 222-231, 2011.

[63] M. J. Young, D. O’Meally, S. D. Sarre, A. Georges, and T. Ezaz, "Molecular cytogenetic map of the central bearded dragon, Pogona vitticeps (Squamata: Agamidae)," Chromosome Research, vol. 21, no. 4, pp. 361-374, 2013.

[64] A. Kawai, C. Nishida-Umehara, J. Ishijima, Y. Tsuda, H. Ota, and Y. Matsuda, "Different origins of bird and reptile sex chromosomes inferred from comparative mapping of chicken Z-linked genes," Cytogenetic and Genome Research, vol. 117, no. 1-4, pp. 92-102, 2007.

[65] M. Tone, Y. Sakaki, T. Hashiguchi, and S. Mizuno, "Genus specificity and extensive methylation of the $\mathrm{W}$ chromosomespecific repetitive DNA sequences from the domestic fowl, Gallus gallus domesticus," Chromosoma, vol. 89, no. 3, pp. 228237, 1984. 
[66] P. P. Rabenold, W. H. Piper, M. D. Decker, and D. J. Minchella, "Polymorphic minisatellite amplified on avian W chromosome," Genome, vol. 34, no. 3, pp. 489-493, 1991.

[67] C. Y. Miyaki, O. Hanotte, A. Wajntal, and T. Burke, "Sex typing of Aratinga parrots using the human minisatellite probe 33.15," Nucleic Acids Research, vol. 20, no. 19, pp. 5235-5236, 1992.

[68] M. E. Delany, T. M. Gessaro, K. L. Rodrigue, and L. M. Daniels, "Chromosomal mapping of chicken mega-telomere arrays to GGA9, 16, 28 and W using a cytogenomic approach," Cytogenetic and Genome Research, vol. 117, no. 1-4, pp. 54-63, 2007.

[69] P. Forster, A. Röhl, P. Lünnemann et al., "A short tandem repeatbased phylogeny for the human Y chromosome," The American Journal of Human Genetics, vol. 67, no. 1, pp. 182-196, 2000.

[70] A. J. Macdonald, N. Sankovic, S. D. Sarre et al., "Y chromosome microsatellite markers identified from the tammar wallaby (Macropus eugenii) and their amplification in three other macropod species," Molecular Ecology Notes, vol. 6, no. 4, pp. 1202-1204, 2006.

[71] V. J. Murtagh, D. O'Meally, N. Sankovic et al., "Evolutionary history of novel genes on the tammar wallaby Y chromosome: implications for sex chromosome evolution," Genome Research, vol. 22, no. 3, pp. 498-507, 2012.

[72] R. D. Kortschak, E. Tsend-Ayush, and F. Grützner, "Analysis of SINE and LINE repeat content of Y chromosomes in the platypus, Ornithorhynchus anatinus," Reproduction, Fertility and Development, vol. 21, no. 8, pp. 964-975, 2009.

[73] D. Bachtrog, "Y-chromosome evolution: emerging insights into processes of Y-chromosome degeneration," Nature Reviews Genetics, vol. 14, no. 2, pp. 113-124, 2013.

[74] A. B. Carvalho and A. G. Clark, "Y chromosome of D. pseudoobscura is not homologous to the ancestral Drosophila Y,' Science, vol. 307, no. 5706, pp. 108-110, 2005.

[75] Q. Zhou and D. Bachtrog, "Sex-specific adaptation drives early sex chromosome evolution in Drosophila," Science, vol. 337, no. 6092, pp. 341-345, 2012.

[76] Q. Zhou, C. E. Ellison, V. B. Kaiser, A. A. Alekseyenko, A. A. Gorchakov, and D. Bachtrog, "The epigenome of evolving Drosophila neo-sex chromosomes: dosage compensation and heterochromatin formation," PLoS Biology, vol. 11, no. 11, Article ID e1001711, 2013.

[77] D. Bachtrog, E. Hom, K. M. Wong, X. Maside, and P. de Jong, "Genomic degradation of a young Y chromosome in Drosophila miranda," Genome Biology, vol. 9, no. 2, article R30, 2008.

[78] M. Steinemann and S. Steinemann, "Degenerating Y chromosome of Drosophila miranda: a trap for retrotransposons," Proceedings of the National Academy of Sciences of the United States of America, vol. 89, no. 16, pp. 7591-7595, 1992.

[79] L. Singh, I. F. Purdom, and K. W. Jones, "Satellite DNA and evolution of sex chromosomes," Chromosoma, vol. 59, no. 1, pp. 43-62, 1976.

[80] J. Arnemann, S. Jakubiczka, J. Schmidtke, R. Schäfer, and J. T. Epplen, "Clustered GATA repeats (Bkm sequences) on the human Y chromosome," Human Genetics, vol. 73, no. 4, pp. 301303, 1986.

[81] K. W. Jones and L. Singh, "Conserved repeated DNA sequences in vertebrate sex chromosomes," Human Genetics, vol. 58, no. 1, pp. 46-53, 1981.

[82] I. Nanda, C. Deubelbeiss, M. Guttenbach, J. T. Epplen, and M. Schmid, "Heterogeneities in the distribution of (GACA)n simple repeats in the karyotypes of primates and mouse," Human Genetics, vol. 85, no. 2, pp. 187-194, 1990.
[83] I. Nanda, H. Zischler, C. Epplen, M. Guttenbach, and M. Schmid, "Chromosomal organization of simple repeated DNA sequences used for DNA fingerprinting," Electrophoresis, vol. 12, no. 2-3, pp. 193-203, 1991.

[84] D. O’Meally, H. R. Patel, R. Stiglec et al., "Non-homologous sex chromosomes of birds and snakes share repetitive sequences," Chromosome Research, vol. 18, no. 7, pp. 787-800, 2010.

[85] L. Singh, I. F. Purdom, and K. W. Jones, "Conserved sexchromosome-associated nucleotide sequences in eukaryotes," Cold Spring Harbor Symposia on Quantitative Biology, vol. 45, part 2, pp. 805-814, 1981.

[86] C. Biémont and C. Vieira, "Genetics: junk DNA as an evolutionary force," Nature, vol. 443, no. 7111, pp. 521-524, 2006.

[87] S. Ohno, "So much "junk" DNA in our genome," Brookhaven Symposia in Biology, vol. 23, pp. 366-370, 1972.

[88] N. Chen, D. W. Bellott, D. C. Page, and A. G. Clark, "Identification of avian W-linked contigs by short-read sequencing," BMC Genomics, vol. 13, no. 1, article 183, 2012.

[89] J. M. J. Lepesant, C. Cosseau, J. Boissier et al., "Chromatin structural changes around satellite repeats on the female sex chromosome in Schistosoma mansoni and their possible role in sex chromosome emergence," Genome Biology, vol. 13, no. 2, article R14, 2012.

[90] T. B. Sackton and D. L. Hartl, "Meta-analysis reveals that genes regulated by the $\mathrm{Y}$ chromosome in Drosophila melanogaster are preferentially localized to repressive chromatin," Genome Biology and Evolution, vol. 5, no. 1, pp. 255-266, 2013.

[91] P. P. Jiang, D. L. Hartl, and B. Lemos, "Y not a dead end: epistatic interactions between Y-linked regulatory polymorphisms and genetic background affect global gene expression in Drosophila melanogaster," Genetics, vol. 186, no. 1, pp. 109-118, 2010.

[92] B. Lemos, L. O. Araripe, and D. L. Hartl, "Polymorphic Y chromosomes harbor cryptic variation with manifold functional consequences," Science, vol. 319, no. 5859, pp. 91-93, 2008.

[93] B. Lemos, A. T. Branco, and D. L. Hartl, "Epigenetic effects of polymorphic Y chromosomes modulate chromatin components, immune response, and sexual conflict," Proceedings of the National Academy of Sciences of the United States of America, vol. 107, no. 36, pp. 15826-15831, 2010.

[94] T. B. Sackton, H. Montenegro, D. L. Hartl, and B. Lemos, "Interspecific Y chromosome introgressions disrupt testisspecific gene expression and male reproductive phenotypes in Drosophila," Proceedings of the National Academy of Sciences of the United States of America, vol. 108, no. 41, pp. 17046-17051, 2011.

[95] J. H. Malone and B. Oliver, "The sex chromosome that refused to die," BioEssays, vol. 30, no. 5, pp. 409-411, 2008.

[96] W. R. Rice and U. Friberg, "Genetics: Functionally degenerate Y not so?” Science, vol. 319, no. 5859, pp. 42-43, 2008.

[97] J. Zhou, T. B. Sackton, L. Martinsen, B. Lemos, T. H. Eickbush, and D. L. Hartl, "Y chromosome mediates ribosomal DNA silencing and modulates the chromatin state in Drosophila," Proceedings of the National Academy of Sciences of the United States of America, vol. 109, no. 25, pp. 9941-9946, 2012.

[98] R. Piergentili, "Multiple roles of the Y chromosome in the biology of Drosophila melanogaster," TheScientificWorldJournal, vol. 10, pp. 1749-1767, 2010.

[99] L. K. Case, E. H. Wall, J. A. Dragon et al., "The y chromosome as a regulatory element shaping immune cell transcriptomes and susceptibility to autoimmune disease," Genome Research, vol. 23, no. 9, pp. 1474-1485, 2013. 
[100] N. P. Singh, S. R. Madabhushi, S. Srivastava et al., "Epigenetic profile of the euchromatic region of human y chromosome," Nucleic Acids Research, vol. 39, no. 9, pp. 3594-3606, 2011.

[101] P. Priyadarshini, B. S. Murthy, J. Nagaraju, and L. Singh, "A GATA-binding protein expressed predominantly in the pupal ovary of the silkworm, Bombyx mori," Insect Biochemistry and Molecular Biology, vol. 33, no. 2, pp. 185-195, 2003.

[102] L. Singh, R. Wadhwa, S. Naidu, R. Nagaraj, and M. Ganesan, "Sex- and tissue-specific Bkm(GATA)-binding protein in the germ cells of heterogametic sex," Journal of Biological Chemistry, vol. 269, no. 41, pp. 25321-25327, 1994.

[103] S. Subramanian, R. K. Mishra, and L. Singh, "Genome-wide analysis of Bkm sequences (GATA repeats): Predominant association with sex chromosomes and potential role in higher order chromatin organization and function," Bioinformatics, vol. 19, no. 6, pp. 681-685, 2003.

[104] R. P. Kumar, J. Krishnan, N. Pratap Singh, L. Singh, and R. K. Mishra, "GATA simple sequence repeats function as enhancer blocker boundaries," Nature Communications, vol. 4, article 1844, 2013. 

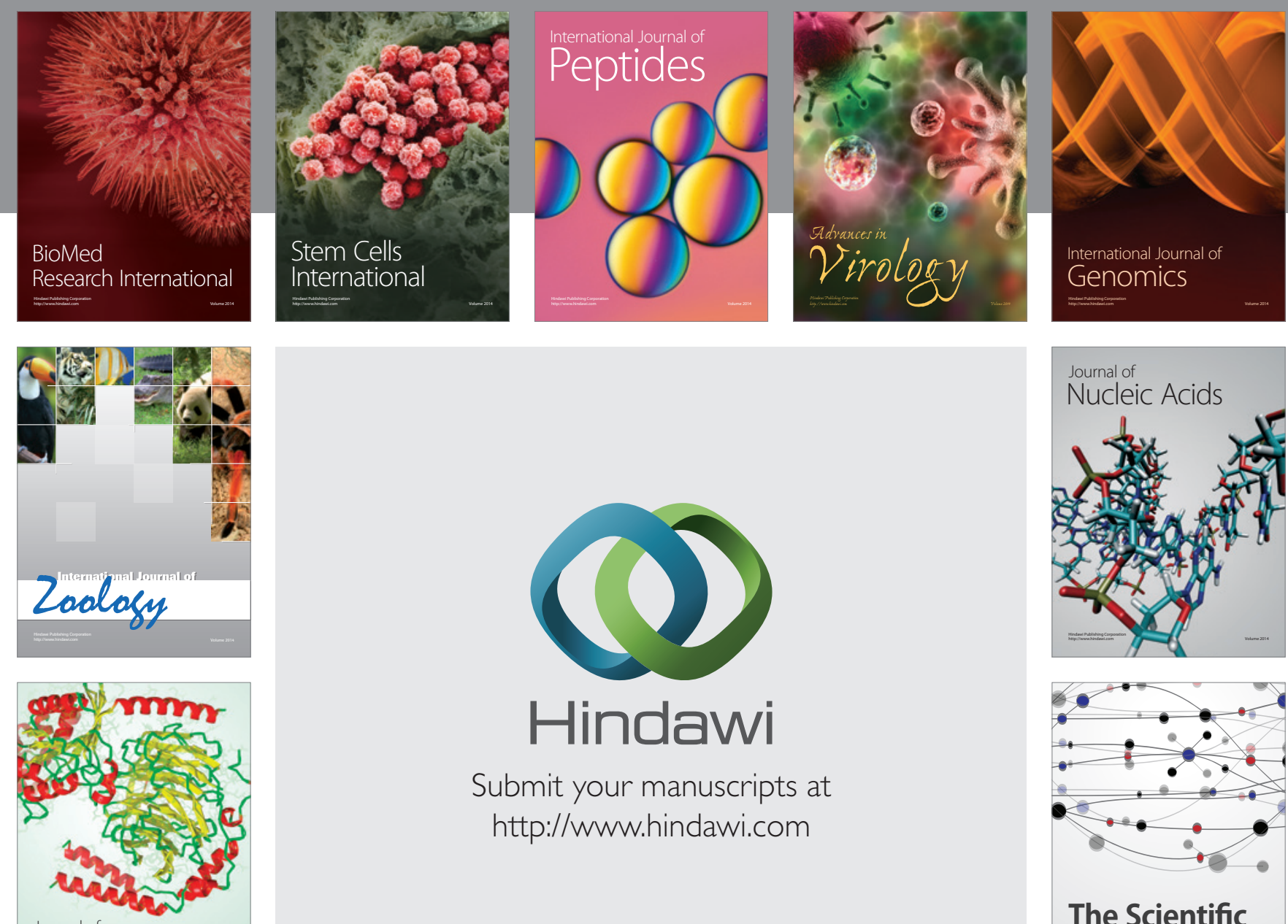

Submit your manuscripts at

http://www.hindawi.com

Journal of
Signal Transduction
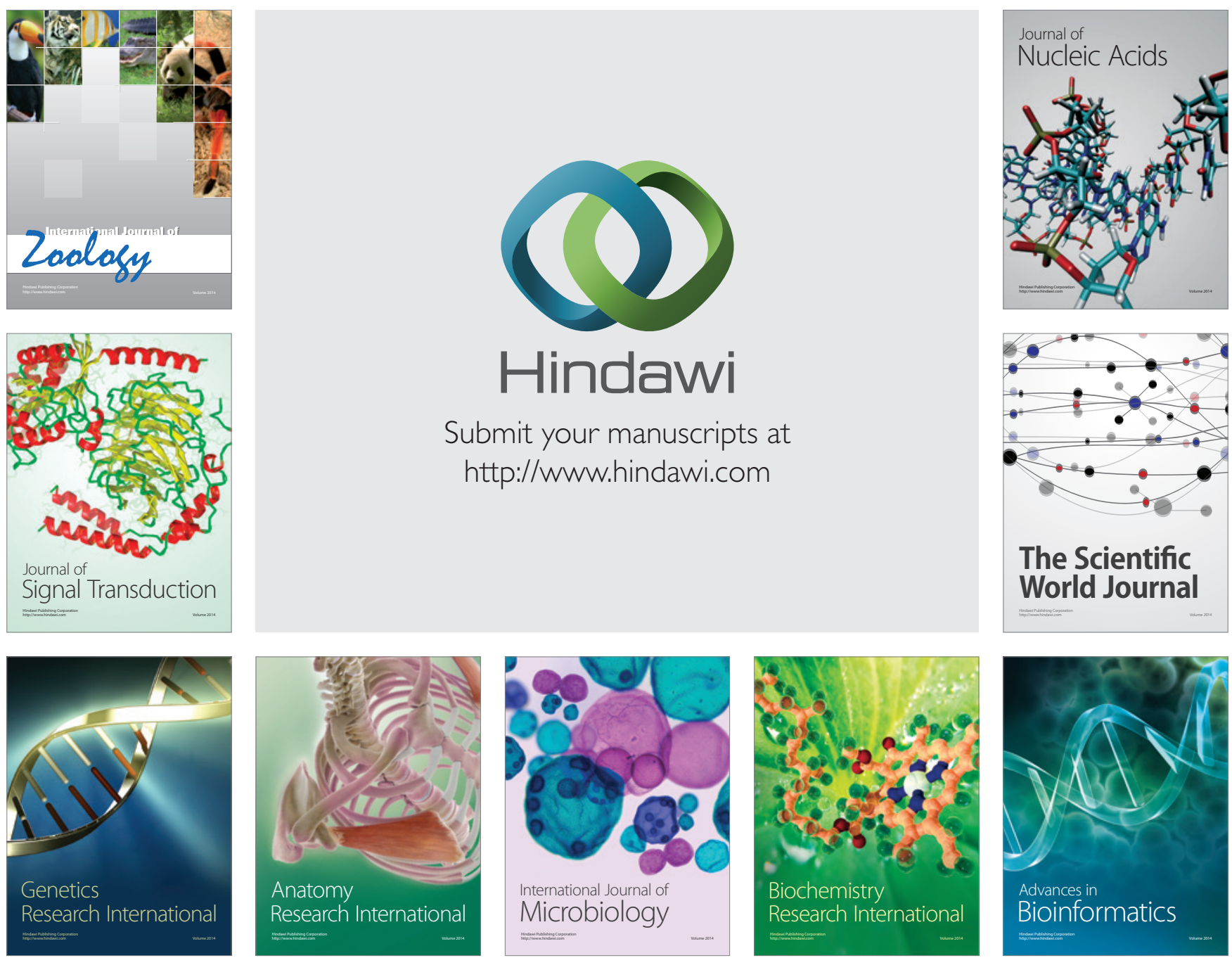

The Scientific World Journal
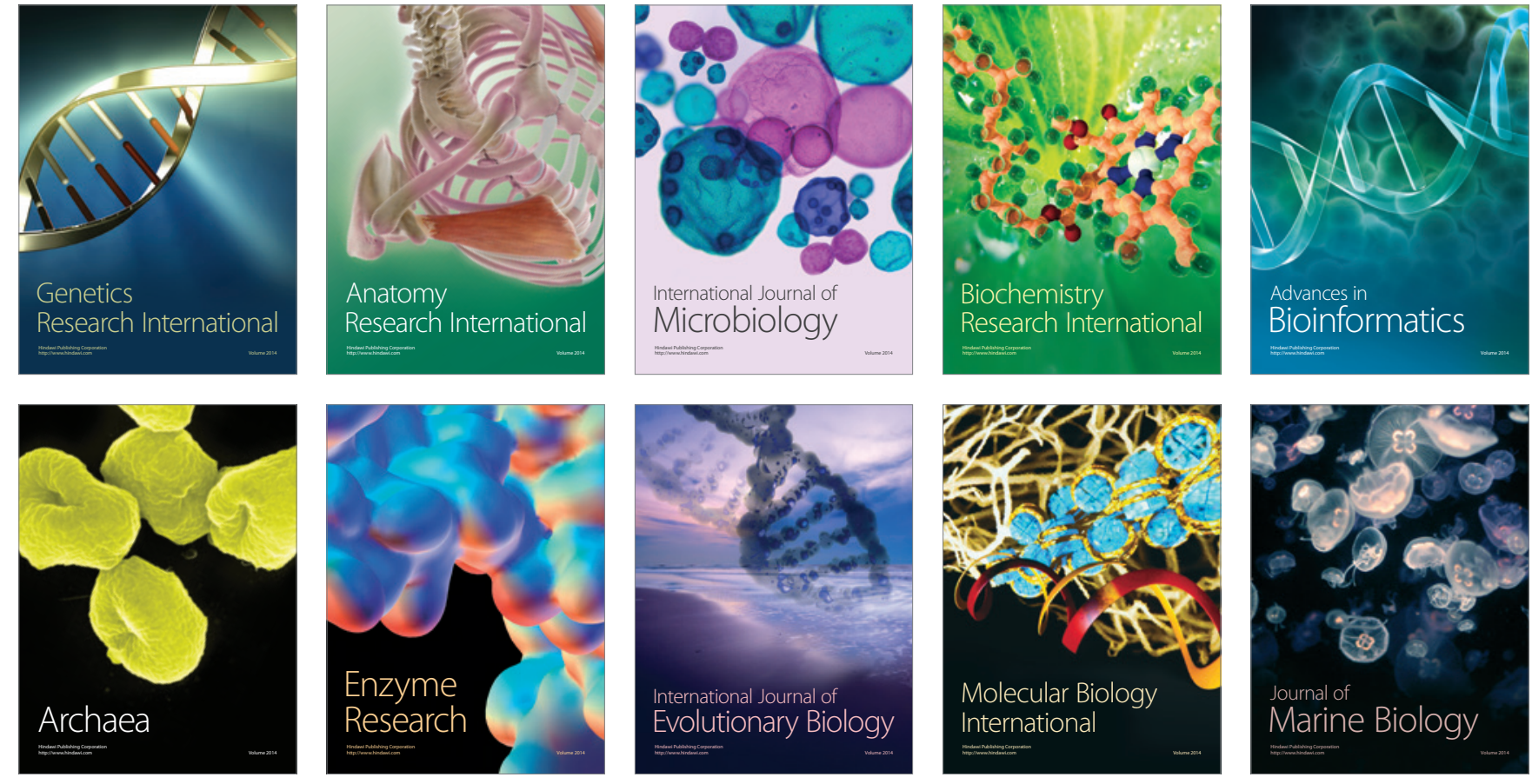